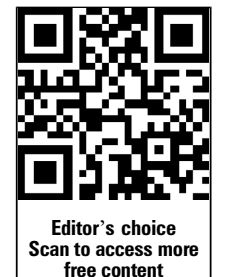

free content
${ }^{1}$ Division of Gastroenterology \& Hepatology, Department of Internal Medicine III, Medical University of Vienna, Vienna, Austria

${ }^{2}$ Division of Gastroenterology \& Hepatology, Department of Internal Medicine I, Hospital Hietzing, Vienna, Austria

\section{Correspondence to}

Professor Dr Markus Peck-

Radosavljevic, Department of Internal Medicine III, Division of Gastroenterology \& Hepatology, Vienna Hepatic Hemodynamic Laboratory, Medical University Vienna, Waehringer Guertel 18-20, A-1090 Vienna, Austria; markus.peck@meduniwien.ac.at

Received 30 October 2012 Revised 22 November 2012 Accepted 23 November 2012 Published Online First 18 December 2012

\section{SLinked}

- http://dx.doi.org/10.1136/ gutjnl-2012-304182

To cite: Reiberger $T$ Ulbrich G, Ferlitsch A, et al. Gut 2013;62:1634-1641.

\title{
Carvedilol for primary prophylaxis of variceal bleeding in cirrhotic patients with haemodynamic non-response to propranolol
}

\author{
Thomas Reiberger, ${ }^{1}$ Gregor Ulbrich, ${ }^{2}$ Arnulf Ferlitsch, ${ }^{1}$ Berit Anna Payer, ${ }_{1}$ \\ Philipp Schwabl, ${ }^{1}$ Matthias Pinter, ${ }^{1}$ Birgit B Heinisch, ${ }^{1}$ Michael Trauner, ${ }^{1}$ \\ Ludwig Kramer, ${ }^{2}$ Markus Peck-Radosavljevic, ${ }^{1}$ Vienna Hepatic Hemodynamic Lab
}

\section{ABSTRACT}

Objective Non-selective $\beta$-blockers or endoscopic band ligation $(\mathrm{EBL})$ are recommended for primary prophylaxis of variceal bleeding in patients with oesophageal varices. Additional $\alpha$-adrenergic blockade (as by carvedilol) may increase the number of patients with haemodynamic response (reduction in hepatic venous pressure gradient (HVPG) of $\geq 20 \%$ or to values $<12 \mathrm{~mm} \mathrm{Hg}$ ).

Design Patients with oesophageal varices undergoing measurement of HVPG before and under propranolol treatment (80-160 mg/day) were included. HVPG responders were kept on propranolol (PROP group), while non-responders were placed on carvedilol (6.25$50 \mathrm{mg} /$ day). Carvedilol responders continued treatment (CARV group), while non-responders to carvedilol underwent EBL. The primary aim was to assess haemodynamic response rates to carvedilol in propranolol non-responders.

Results 36\% (37/104) of patients showed a HVPG response to propranolol. Among the propranolol nonresponders $56 \%$ (38/67) eventually achieved a haemodynamic response with carvedilol, while 44\% (29/67) patients were finally treated with EBL. The decrease in HVPG was significantly greater with carvedilol (median $12.5 \mathrm{mg} /$ day) than with propranolol (median $100 \mathrm{mg} / \mathrm{day}$ ): $-19 \pm 10 \%$ versus $-12 \pm 11 \%$ $(p<0.001)$. During a 2 year follow-up bleeding rates for PROP were $11 \%$ versus CARV $5 \%$ versus EBL $25 \%$ $(p=0.0429)$. Fewer episodes of hepatic decompensation (PROP 38\%/CARV 26\% vs EBL 55\%; $p=0.0789$ ) and significantly lower mortality (PROP 14\%/CARV 11\% vs $\mathrm{EBL} 31 \% ; p=0.0455)$ were observed in haemodynamic responders compared to the EBL group.

Conclusions Carvedilol leads to a significantly greater decrease in HVPG than propranolol. Using carvedilol for primary prophylaxis a substantial proportion of nonresponders to propranolol can achieve a haemodynamic response, which is associated with improved outcome with regard to prevention of variceal bleeding, hepatic decompensation and death.

\section{INTRODUCTION}

Portal hypertension (PHT) is responsible for the majority of complications of patients with cirrhotic of the liver, for example, the development of oesophageal varices, ascites, hepatorenal syndrome, hyperdynamic circulation and hepatic encephalopathy. ${ }^{1}{ }^{2}$

\section{Significance of this study}

What is already known on this subject?

- Pilot trials have shown that carvedilol, a NSBB with additional anti- $\alpha_{1}$-adrenergic activity may decrease portal pressure more effectively than propranolol.

- A randomised controlled trial comparing carvedilol to EBL for primary prophylaxis of variceal bleeding showed fewer bleeding events in the carvedilol group.

- Carvedilol may cause arterial hypotension and worsen renal function potentially compromising its beneficial effect in the long term.

What are the new findings?

- Carvedilol is effective in a substantial proportion of patients who did not achieve a haemodynamic response to propranolol.

- The mean decrease of portal pressure is significantly more pronounced under carvedilol $(-19 \%)$ than under propranolol $(-12 \%)$.

- Higher doses of carvedilol (>12.5 mg/day) may not further decrease portal pressure, while increasing the risk of arterial hypotension and bradycardia.

- A long-term follow-up of up to 2 years is reported even showing a benefit in terms of reduced incidence of hepatic decompensation and improved survival.

How might it impact on clinical practice in the foreseeable future?

- Carvedilol may replace propranolol as a NSBB without anti- $\alpha_{1}$-adrenergic activity in primary prophylaxis of variceal bleeding - at least in patients without arterial hypotension or renal dysfunction.

- Carvedilol doses of 6.25-12.5 mg/day might be adequate for primary prophylaxis of variceal bleeding.

- A haemodynamic response to $\beta$-blockers is associated with a lower risk of hepatic decompensation and mortality.

- The efficacy of EBL for primary prophylaxis in non-responders to $\beta$-blockers is limited and other options such as transjugular intrahepatic portosystemic shunt implantation should be investigated in future trials. 
Patients with oesophageal varices are at risk of variceal bleeding, especially if varices are large or present red spot signs. ${ }^{3}{ }^{4}$ Despite advances in the management of acute variceal bleeding the inhospital mortality is still as high as $20 \% .^{5}$

Earlier studies have already shown that achieving a haemodynamic response to non-selective $\beta$-blockers (NSBB) (defined as a decrease in hepatic venous pressure gradient (HVPG) of $\geq 20 \%$ compared to baseline values or to absolute values $<12 \mathrm{~mm} \mathrm{Hg}$ ) treatment may protect from variceal bleeding. ${ }^{6-8}$ Consequently, current guidelines ${ }^{9}$ suggest pharmacological treatment with NSBB or endoscopic band ligation (EBL) for the prevention of the first variceal bleeding episode. Nadolol and propranolol have traditionally been used for prophylaxis of variceal bleeding, ${ }^{10-13}$ while carvedilol represents a promising drug that needs to be explored further. ${ }^{9}$ Carvedilol is a racemic mixture that possesses both non-selective $\beta_{1 / 2}$-antagonist and $\alpha_{1}$-receptor antagonist activity. ${ }^{14}$ Given its combined mechanism of action, carvedilol may have a greater potential for lowering portal pressure than propranolol. ${ }^{15} 16$

The haemodynamic response to carvedilol has already been assessed in earlier studies: A small pilot study in 16 patients ${ }^{17}$ demonstrated a fall in HVPG from 16.7 to $13.6 \mathrm{~mm} \mathrm{Hg}$ without a significant reduction in azygos blood flow. Mean arterial pressure (MAP) dropped from 94.8 to $84 \mathrm{~mm} \mathrm{Hg}$, while heart rate decreased only in patients with ascites. No changes in cardiac output, renal vein blood flow, or systemic vascular resistance were observed.

A randomised trial comparing the acute administration of carvedilol to propranolol has shown a more effective reduction in portal pressure with carvedilol than with propranolol, while carvedilol caused a more pronounced fall in MAP. ${ }^{18}$ Arterial hypotension caused by carvedilol may eventually prevent its long-term use in cirrhotic patients with hyperdynamic circulation and impaired renal function. The efficacy and safety of the long-term use of carvedilol compared to propranolol in portal hypertensive patients was addressed in another study, ${ }^{19}$ showing that the proportion of patients achieving a haemodynamic response was greater with carvedilol. After a median observation period of 11 weeks, carvedilol caused a significant decrease in MAP, a significant increase in plasma volume and body weight. While the glomerular filtration rate was unchanged with carvedilol, doses of diuretics were increased more frequently in the carvedilol group. ${ }^{19}$

The only long-term randomised trial using carvedilol for primary prophylaxis of variceal bleeding compared with EBL demonstrated significantly lower bleeding rates under carvedilol than with EBL treatment. ${ }^{20}$ However, a fixed dose of carvedilol $(12.5 \mathrm{mg} /$ day) was used and haemodynamic response to carvedilol has not been evaluated by HVPG in that trial. ${ }^{20}$

Despite these promising data on the use of carvedilol, no data on the efficacy of carvedilol in patients not responding to propranolol-as assessed by repeated HVPG measurements-are available. As pointed out by some experts ${ }^{14} 19$ and the current guidelines, ${ }^{9}$ further data on the long-term efficacy of carvedilol for primary prophylaxis of variceal bleeding are needed.

Therefore, the primary aim of our study on primary prophylaxis of variceal bleeding was to evaluate haemodynamic response rates to carvedilol in propranolol non-responders.

Secondary aims were: (1) to compare the antiportal hypertensive effects of carvedilol to propranolol; (2) to assess the systemic haemodynamic effects of carvedilol and propranolol; (3) to evaluate the incidence of variceal bleeding during primary prophylaxis of variceal bleeding; and (4) to report the rates of hepatic decompensation and mortality during long-term follow-up in propranolol, carvedilol, and EBL-treated patients.

\section{PATIENTS AND METHODS}

\section{Patients}

This study was conducted from 2008 to 2012 following the ethical principles of the revised Helsinki Declaration and was approved by the local ethics committee. Patients with cirrhotic referred for haemodynamic evaluation of PHT were eligible for the study. Inclusion criteria were the presence of oesophageal varices at upper gastrointestinal endoscopy ${ }^{21}$ without a previous haemorrhage and a basal HVPG value greater than $12 \mathrm{~mm} \mathrm{Hg}$. Exclusion criteria were as following: age less than 18 years, pre or posthepatic causes of PHT, severe liver failure (serum bilirubin $>5 \mathrm{mg} / \mathrm{dl}$ or international normalised ratio $($ INR) $>2.5$ ), uncontrolled hepatic encephalopathy, alcohol consumption, or intravenous drug abuse, renal insufficiency (serum creatinine $>1.5 \mathrm{mg} / \mathrm{dl}$ ), contraindications to NSBB, a diagnosis of hepatocellular carcinoma (HCC) or other malignancy, or refusal to participate in the study. Patients with cirrhotic due to alcoholic liver disease had to be abstinent for at least 3 months before inclusion, which was evaluated by clinic visits every 4 weeks. All patients gave their written informed consent to participate in the study and to the publication of their anonymised data.

\section{Treatment and dosing of NSBB}

The dose of NSBB was titrated according to systolic arterial blood pressure $\left(\mathrm{ABP}_{\text {sys }}\right)$ and heart rate $(\mathrm{HR})$, as previously described. ${ }^{22}$ Doses were increased weekly until $\mathrm{ABP}_{\text {sys }}$ was not less than $95 \mathrm{~mm} \mathrm{Hg}$ and HR was not less than $50 \mathrm{bpm}$. Patients receiving propranolol (Inderal, Astra Zenica, Austria) started at a dose of $40 \mathrm{mg} / \mathrm{day}$ and the dose was increased weekly (in $40 \mathrm{mg}$ steps) according to $\mathrm{ABP}_{\text {sys }}$ and $\mathrm{HR}$ to a maximum dose of $160 \mathrm{mg} /$ day. By retrospective analysis of haemodynamic data recorded in our haemodynamic laboratory, propranolol target doses of $80-160 \mathrm{mg} /$ day were chosen, as the haemodynamic efficacy of propranolol doses less than $80 \mathrm{mg}$ /day was poor $(<10 \%$ haemodynamic response) and only $3 \%$ of patients could tolerate (eg, vertigo, nausea, headache) propranolol doses greater than $160 \mathrm{mg} /$ day. Carvedilol (Dilatrend, Hoffmann La-Roche, Vienna, Austria) was started at a dose of $6.25 \mathrm{mg} /$ day and titrated to a maximum dose of $25 \mathrm{mg}$ /day by steps of $6.25 \mathrm{mg}$ per week (except in patients with concomitant arterial hypertension, who were started with $12.5 \mathrm{mg} /$ day and increased up to $50 \mathrm{mg} /$ day). Compliance with therapy was monitored by monitoring of $\mathrm{HR}$ and blood pressure during clinical visits.

\section{Sample size calculation and study design}

After baseline haemodynamic assessment, treatment with propranolol was initiated and titrated according to $\mathrm{ABP}_{\text {sys }}$ and $\mathrm{HR}$ (figure 1). After 4 weeks a second HVPG measurement was performed under the maximal tolerated dose of propranolol and the haemodynamic response was evaluated. Haemodynamic response was defined as a decrease of HVPG of more than 20\% compared to baseline or a decrease to absolute values less than $12 \mathrm{~mm} \mathrm{Hg}^{7}{ }^{9}$ Haemodynamic responders to propranolol were kept on propranolol (PROP group), while haemodynamic nonresponders were then switched to carvedilol with weekly increasing doses until the maximum tolerated dose. If patients were intolerant to propranolol or were not able to increase the dose of propranolol to $80 \mathrm{mg} /$ day or greater, they were directly started with carvedilol. There was no wash-out phase between propranolol and carvedilol treatment. In patients switched to carvedilol, a third HVPG measurement was performed after 4 weeks of carvedilol treatment to evaluate the haemodynamic response to carvedilol. Again haemodynamic responders to 
Figure 1 Patient flow chart. Among 173 patients screened, 131 patients were study candidates. After excluding patients with HCC, portal vein thrombosis, renal insufficiency and patients who refused to participate in the study protocol, a total number of 104 cirrhotic patients was included. $E B L$, endoscopic band ligation; HCC, hepatocellular carcinoma; HVPG, hepatic venous pressure gradient.

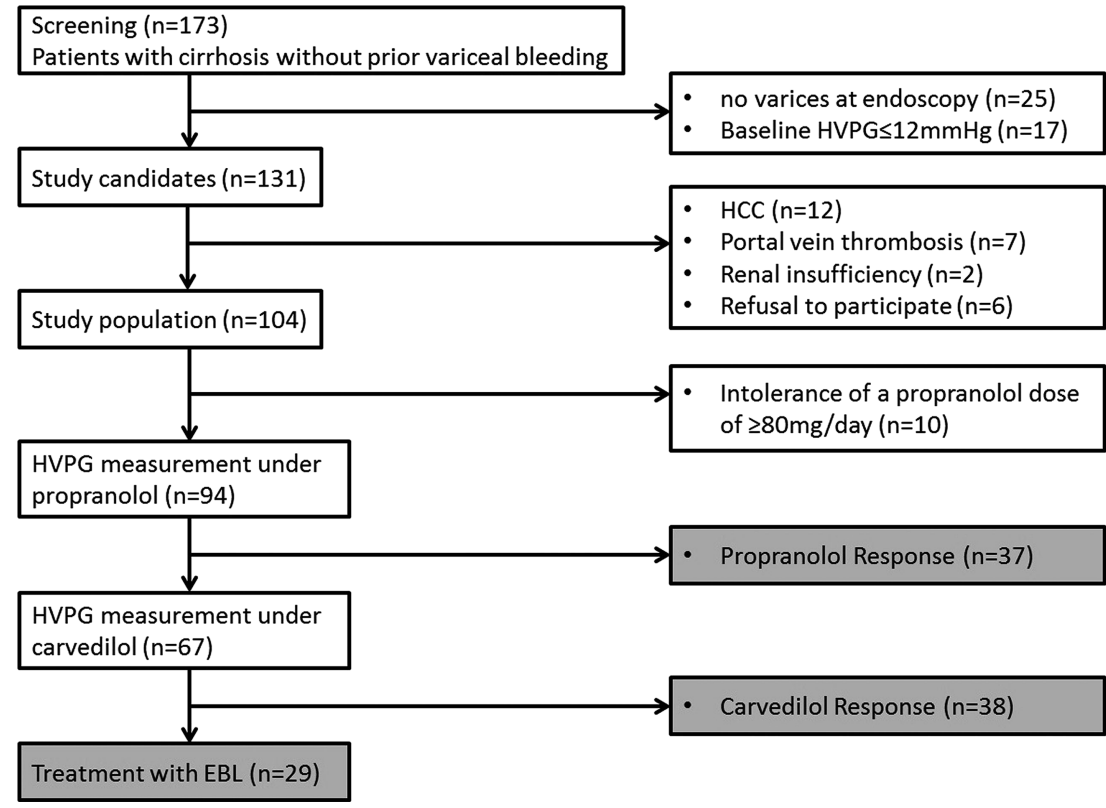

carvedilol were kept on carvedilol (CARV group), while carvedilol non-responders underwent EBL every 2-4 weeks until eradication of varices. After eradication of varices, endoscopy was repeated every 6 months to re-evaluate the presence of varices and eventually to perform additional band ligations. According to this protocol, three groups entered the follow-up period:

1. PROP group: patients with haemodynamic response to propranolol.

2. CARV group: patients with haemodynamic response to carvedilol.

3. EBL group: patients not responding/intolerant to propranolol/carvedilol as evaluated by HVPG and who were treated by EBL until eradication of varices.

One hundred and fourteen patients had to be included to guarantee a sample size of 68 patients needed for the primary analysis, assuming a propranolol response rate of $40 \%$. A sample size of 68 patients had a calculated $90 \%$ power to detect a decrease in portal pressure of $2 \mathrm{~mm} \mathrm{Hg}$ with carvedilol in the group of propranolol non-responders, assuming a SD of differences of $5 \mathrm{~mm} \mathrm{Hg}$ and using a paired t test with a 0.05 two-sided significance level.

All patients in the three treatment groups were followed up every 3 months with clinic visits including laboratory testing to evaluate treatment-related side effects, bleeding rates, laboratory abnormalities, renal function and mortality during propranolol, carvedilol and EBL treatment for primary prophylaxis of variceal bleeding. Hepatic decompensation was defined as hepatic encephalopathy grade $3 / 4$, development of ascites, variceal bleeding, or jaundice.

\section{Measurement of HVPG and liver stiffness}

Measurement of HVPG was performed as an outpatient procedure according to reference methodology ${ }^{23}$ as previously described. ${ }^{24}$ Briefly, under ultrasound guidance and local anaesthesia a catheter introducer set was placed in the right internal jugular vein by using the Seldinger technique. A balloon catheter was introduced over the upper and lower inferior caval vein by passing the heart into a large liver vein. Correct placement and sufficient wedge position were checked by x-ray. At least three repeated measurements of free and wedged hepatic vein pressure were performed to calculate HVPG. Pressure curves were continuously recorded via a haemodynamic interface connected to a PC and by using licensed software (S5 Collect, Vienna, Austria) for subsequent print-out and evaluation. Liver stiffness was measured by transient elastography (Fibroscan, Echosens, France) as previously described. ${ }^{25}$

\section{Statistical analysis}

Continuous variables were expressed as mean \pm SD. Categorical variables were expressed as median and range. Comparison between baseline and follow-up haemodynamic variables were computed using the paired Student's $t$ test. The decrease in HVPG by propranolol or carvedilol was calculated in relation to the baseline HVPG assessment, respectively. The haemodynamic effects of propranolol and carvedilol were compared using the non-parametric Mann-Whitney U test. The significance levels between the proportions were calculated by Fisher's exact test or the $\chi^{2}$ test. Rates of variceal bleeding and hepatic decompensation, and transplant-free survival (TFS) were calculated by Kaplan-Meier analysis and compared by log-rank test. A p value of less than 0.05 was considered to denote statistical significance.

\section{RESULTS}

\section{Patient characteristics}

Within the study period 173 cirrhotic patients without a history of variceal haemorrhage were referred for evaluation of PHT (table 1). Among those patients, 25 did not show oesophageal varices at endoscopy and 17 patients had a baseline HVPG value of $12 \mathrm{~mm} \mathrm{Hg}$ or less. Twenty-one patients met the exclusion criteria of the study protocol (HCC, 12; portal vein thrombosis, seven; renal insufficiency, two), while six patients refused to participate in the study. Finally, a total of 104 cirrhotic patients with oesophageal varices and a baseline HVPG greater than $12 \mathrm{~mm} \mathrm{Hg}$ were included in the study protocol. The majority of patients were men (77\%) with a mean age of 53 years. The main aetiologies of cirrhotic were alcoholic $(55 \%)$ or viral liver disease (33\%). Sixty-six, 30, and eight patients presented with Child-Pugh stages A, B and C, respectively. Forty-one patients showed small varices at upper gastrointestinal 
Table 1 Patient characteristics

\begin{tabular}{|c|c|c|c|c|c|}
\hline Patients (n) & $\begin{array}{l}\text { All } \\
104\end{array}$ & $\begin{array}{l}\text { Propranolol responder } \\
37(35.6 \%)\end{array}$ & $\begin{array}{l}\text { Carvedilol responder } \\
38(36.5 \%)\end{array}$ & $\begin{array}{l}\text { Non-responder (EBL group) } \\
29(27.9 \%)\end{array}$ & p Value* \\
\hline Age (years) & $53 \pm 12$ & $52 \pm 10$ & $55 \pm 11$ & $50 \pm 14$ & 0.242 \\
\hline Sex (m/f, \% male) & $80 / 24(77)$ & $29 / 12(78)$ & $28 / 10(74)$ & 23/6 (79) & 0.836 \\
\hline Alcohol (n, \%) & $57(55)$ & $17(46)$ & $26(68)$ & $14(48)$ & 0.214 \\
\hline Viral $(n, \%)$ & $34(33)$ & $14(38)$ & $9(24)$ & $11(38)$ & 0.193 \\
\hline NASH $(n, \%)$ & $11(10)$ & $6(16)$ & $3(8)$ & $2(7)$ & 0.319 \\
\hline Other $(n, \%)$ & $2(2)$ & $0(-)$ & $0(-)$ & $2(7)$ & $\mathrm{N} / \mathrm{A}$ \\
\hline Child-Pugh stage $(\mathrm{A} / \mathrm{B} / \mathrm{C}, \% \mathrm{~B} / \mathrm{C})$ & $66 / 30 / 8(37)$ & 24/9/4 (35) & 26/10/2 (32) & $16 / 11 / 2(45)$ & 0.488 \\
\hline Previous ascites (n, \%) & $11(10)$ & $4(11)$ & $3(8)$ & $4(13)$ & 0.410 \\
\hline MELD (score) & $11.6 \pm 3.0$ & $11.2 \pm 2.8$ & $11.2 \pm 2.6$ & $12.5 \pm 3.7$ & 0.593 \\
\hline Haemoglobin ( $g / d l)$ & $11.6 \pm 1.9$ & $11.6 \pm 1.8$ & $11.5 \pm 1.7$ & $11.9 \pm 2.2$ & 0.668 \\
\hline Platelet count (G/L) & $113 \pm 66$ & $110 \pm 78$ & $114 \pm 59$ & $115 \pm 63$ & 0.778 \\
\hline WBC (G/L) & $5.3 \pm 2.6$ & $5.1 \pm 2.8$ & $5.4 \pm 2.2$ & $5.4 \pm 3.0$ & 0.723 \\
\hline Serum bilirubin (mg/dl) & $2.12 \pm 1.6$ & $1.78 \pm 0.83$ & $2.16 \pm 1.50$ & $2.51 \pm 2.26$ & 0.654 \\
\hline INR & $1.27 \pm 0.17$ & $1.28 \pm 0.21$ & $1.23 \pm 0.14$ & $1.32 \pm 0.13$ & 0.174 \\
\hline Serum albumin (mg/dl) & $35.2 \pm 5.4$ & $35.9 \pm 6.5$ & $34.8 \pm 5.4$ & $34.9 \pm 3.8$ & 0.732 \\
\hline Liver stiffness (kPa) & $44.7 \pm 22.2$ & $42.2 \pm 25.1$ & $43.6 \pm 21.6$ & $49.7 \pm 19.5$ & 0.600 \\
\hline Small varices $<5 \mathrm{~mm}(\mathrm{n}, \%)$ & 41 (39) & $15(41)$ & $17(45)$ & $9(31)$ & 0.519 \\
\hline Large varices $\geq 5 \mathrm{~mm}(\mathrm{n}, \%)$ & $63(61)$ & $22(59)$ & $21(55)$ & $20(69)$ & \\
\hline Presence of red spots $(n, \%)$ & $32(31)$ & $10(27)$ & $15(39)$ & $7(24)$ & 0.338 \\
\hline Baseline HVPG (mm Hg) & $20.5 \pm 4.1$ & $20.8 \pm 4.2$ & $20.4 \pm 4.1$ & $20.2 \pm 3.8$ & 0.703 \\
\hline Heart rate $(\mathrm{bpm})$ & $83 \pm 15$ & $82 \pm 12$ & $86 \pm 16$ & $78 \pm 17$ & 0.166 \\
\hline Mean arterial pressure $(\mathrm{mm} \mathrm{Hg})$ & $101 \pm 14$ & $101 \pm 13$ & $104 \pm 13$ & $96 \pm 14$ & 0.118 \\
\hline
\end{tabular}

endoscopy, 63 patients presented with large varices and 32 patients had red spot signs. At baseline, the mean HVPG was $20.5 \mathrm{~mm} \mathrm{Hg}$ and mean liver stiffness was $44.7 \mathrm{kPa}$.

\section{Haemodynamic response rates and treatment groups}

After baseline HVPG measurement, 104 patients were placed on propranolol (figure 2). While 10 patients did not tolerate propranolol doses of at least $80 \mathrm{mg} /$ day or were generally intolerant to propranolol, 94 patients were evaluated under propranolol treatment. Thirty-nine per cent (37/94) of patients showed a haemodynamic response and were kept on propranolol. Among the 57 propranolol non-responders, 56\% (32/57) achieved a haemodynamic response to carvedilol. All 10 patients who could not be evaluated under sufficient propranolol doses were started with carvedilol and showed good tolerance to carvedilol treatment, with $60 \%(6 / 10)$ showing a haemodynamic response. All together, the haemodynamic response rate to carvedilol was 57\% (38/67). Finally, 28\% (29/104) patients could not achieve a haemodynamic response to NSBB and were treated with EBL until variceal eradication, while $72 \%(75 / 104)$ of patients showed a HVPG-controlled response to pharmacological treatment with propranolol or carvedilol. Thirty-seven, 38 and 29 patients entered the follow-up period on propranolol (PROP), carvedilol (CARV) and EBL treatment (EBL), respectively. No drop-outs were recorded during haemodynamic evaluation, for example, between the baseline HVPG measurement and assessment under carvedilol.

\section{Haemodynamic effects of NSBB}

Mean baseline HVPG values were $20.5 \mathrm{~mm} \mathrm{Hg}$, mean HVPG values under propranolol were $18 \pm 1 \mathrm{~mm} \mathrm{Hg}(\mathrm{p}<0.01$ vs baseline), and $16 \pm 4 \mathrm{~mm} \mathrm{Hg}$ ( $\mathrm{p}<0.01$ vs baseline) under carvedilol treatment, respectively (table 2 and figure 2). The decrease in HVPG was significantly more pronounced in carvedilol-treated $(n=67)$ patients than in propranolol-treated $(n=94)$ patients: $-19 \pm 11 \%$ vs $-12 \pm 12 \%(\mathrm{p}<0.001)$.

In patients with non-response to propranolol a further significant reduction in HVPG of $13 \%$ (19.3 vs $16.6 \mathrm{~mm} \mathrm{Hg}$; $\mathrm{p}<0.01$ ) could be achieved with carvedilol treatment. The mean HVPG values were lower under carvedilol treatment than under propranolol treatment, both in haemodynamic responders $(15.8 \pm 3.2$ vs 15.1 $\pm 3.5 \mathrm{~mm} \mathrm{Hg} ; \mathrm{p}=0.046)$ and in haemodynamic non-responders to pharmacological therapy $(19.3 \pm 3.5$ vs $18.3 \pm 3.3 \mathrm{~mm} \mathrm{Hg}$; $\mathrm{p}=0.031$ ).

Concerning systemic haemodynamics, a significant reduction in MAP was found in patients treated with propranolol $(-11$ $\pm 14 \% ; \mathrm{p}<0.01)$ and carvedilol $(-14 \pm 14 \% ; \mathrm{p}<0.01)$. Similarly, HR significantly decreased under propranolol $(-23 \pm 12 \%$; $\mathrm{p}<0.01)$ and carvedilol $(-17 \pm 15 \% ; \mathrm{p}<0.01)$ treatment. MAP $(88 \pm 14$ vs $83 \pm 14 ; \mathrm{p}=0.452)$ and $\operatorname{HR}(62 \pm 10$ vs $66 \pm 10$; $\mathrm{p}=0.203)$ were similar under propranolol and carvedilol treatment, respectively.

\section{The influence of drug dosing on haemodynamic parameters}

During the 4-week interval between the HVPG measurements, the doses of propranolol and carvedilol were increased according to $\mathrm{HR}, \mathrm{ABP}_{\text {sys }}$ and patient tolerance (table 3 ). The mean dose of propranolol was $97.6 \pm 20.4 \mathrm{mg} /$ day (median $100 \mathrm{mg} /$ day) with 67 patients receiving low-dose $(80-100 \mathrm{mg} /$ day) and 27 patients receiving high-dose (120-160 mg/day) propranolol, respectively. When comparing the doses of propranolol between haemodynamic responders $(99.6 \pm 19.7 \mathrm{mg} /$ day $)$ and non-responders $(93.6 \pm 20.2 \mathrm{mg} /$ day) no significant differences were found $(p=0.612)$. The mean dose of carvedilol was $17.1 \pm 6.8 \mathrm{mg} /$ day 

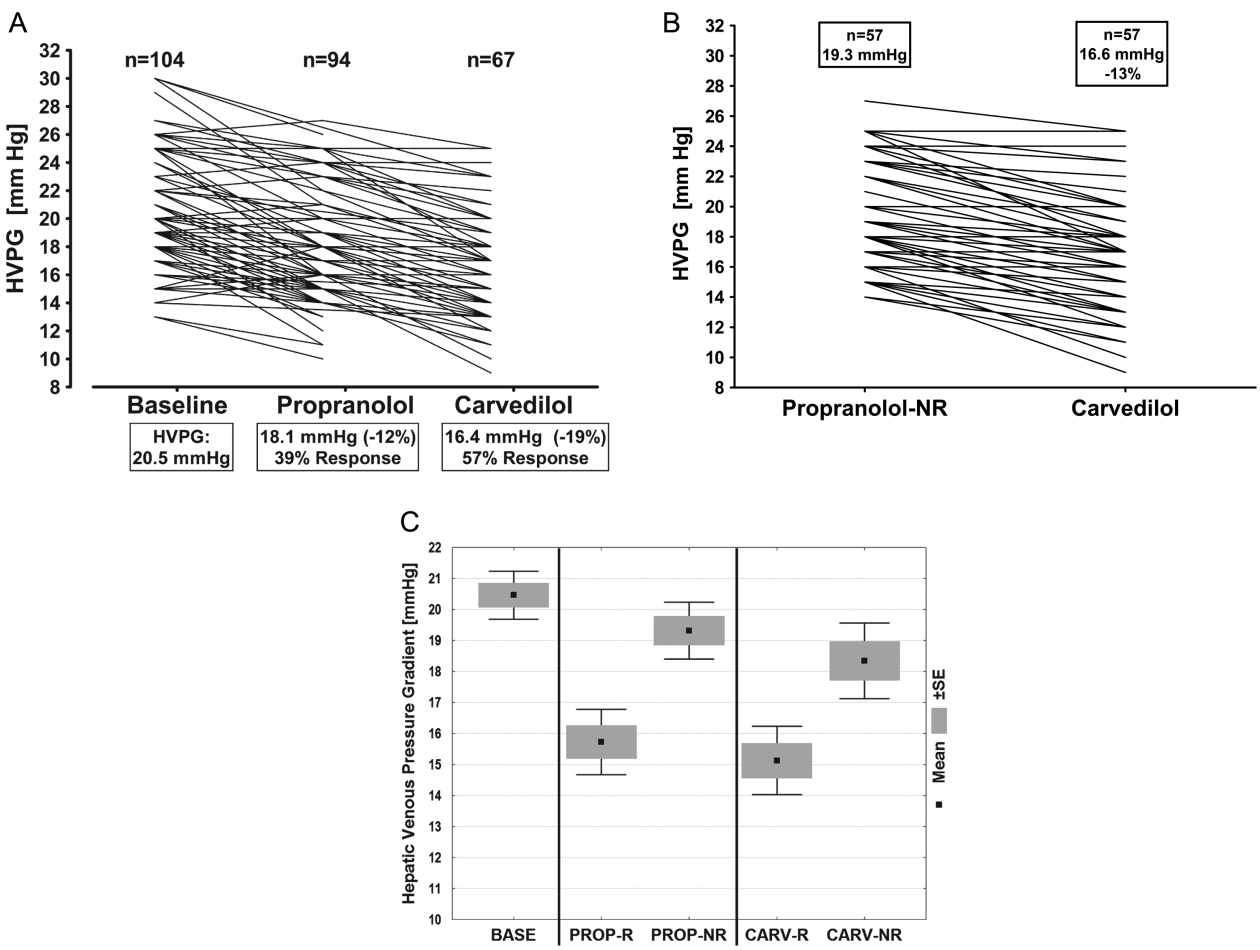

Figure 2 Haemodynamic response to propranol and carvedilol. (A) 104 Patients were evaluated at baseline and showed a mean HVPG of $20.5 \mathrm{~mm} \mathrm{Hg}$. Ninety-four patients were evaluated under propranolol treatment (while 10 were intolerant to a propranolol dose of at least $80 \mathrm{mg} / \mathrm{day}$ ) with $39 \%$ of patients achieving a haemodynamic response to propranolol. The 67 patients with propranolol non-response or intolerance were evaluated under carvedilol and $57 \%$ achieved a haemodynamic response to carvedilol. The mean decrease in HVPG under propranolol and carvedilol was $-12 \%$ and $-19 \%$, respectively, as compared to baseline HVPG. (B) Patients with non-response to propranolol were switched to carvedilol according to the study protocol. Among these 57 patients, a further reduction in HVPG from $19.3 \mathrm{~mm} \mathrm{Hg}$ to $16.6 \mathrm{~mm} \mathrm{Hg}(-13 \%)$ was achieved. (C) This figure shows the mean values of patients at baseline, under propranolol (separately for propranolol responders and non-responders), and under carvedilol (separately for carvedilol responders and non-responders), respectively. A significant reduction in HVPG was found both with propranolol $(p<0.01)$ and with carvedilol $(p<0.01)$. When comparing the subgroups, mean HVPG values were lower under carvedilol treatment than under propranolol treatment, both in haemodynamic responders $(15.8 \pm 3.2 \mathrm{vs} 15.1 \pm 3.5 \mathrm{~mm} \mathrm{Hg} ; \mathrm{p}=0.046)$ and in haemodynamic non-responders to pharmacological therapy $(19.3 \pm 3.5$ vs $18.3 \pm 3.3 \mathrm{~mm} \mathrm{Hg} ; \mathrm{p}=0.031)$. BASE, baseline measurement without $\beta$-blocker therapy; CARV, carvedilol; HVPG, hepatic venous pressure gradient; NR, non-responders; PROP, propranolol; R, responders.

(median $12.5 \mathrm{mg} /$ day) with 40 patients receiving a low dose (6.25-12.5 mg/day) and 27 patients receiving a high dose (25$50 \mathrm{mg} /$ day) of carvedilol, respectively.

Comparing patients with low and high doses of propranolol, no significant differences in MAP $(p=0.328)$, HR $(p=0.106)$ and HVPG $(p=0.930)$ were observed. Patients receiving

Table 2 Haemodynamic effects of propranolol and carvedilol

\begin{tabular}{|c|c|c|c|c|}
\hline & $\begin{array}{l}\text { Baseline } \\
\mathrm{N}=104\end{array}$ & $\begin{array}{l}\text { Propranolol } \\
\mathrm{N}=94\end{array}$ & $\begin{array}{l}\text { Carvedilol } \\
\mathrm{N}=67\end{array}$ & $\begin{array}{l}\text { p Value* propranolol } \\
\text { vs carvedilol }\end{array}$ \\
\hline $\begin{array}{l}\text { HVPG } \\
(\mathrm{mm} \mathrm{Hg})\end{array}$ & $20.5 \pm 4.0$ & $18.1 \pm 3.8$ & $16.4 \pm 2.8$ & $<0.01$ \\
\hline $\begin{array}{l}\text { Change in } \\
\text { HVPG (\%) }\end{array}$ & $\mathrm{N} / \mathrm{A}$ & $-12 \pm 12$ & $-19 \pm 11$ & $<0.01$ \\
\hline $\begin{array}{l}\text { MAP } \\
(\mathrm{mm} \mathrm{Hg})\end{array}$ & $101 \pm 14$ & $88 \pm 14$ & $83 \pm 14$ & 0.457 \\
\hline $\begin{array}{l}\text { Change in } \\
\text { MAP (\%) }\end{array}$ & $\mathrm{N} / \mathrm{A}$ & $-11 \pm 14$ & $-14 \pm 14$ & 0.452 \\
\hline HR (bpm) & $83 \pm 15$ & $62 \pm 10$ & $66 \pm 10$ & 0.203 \\
\hline $\begin{array}{l}\text { Change in } \\
\text { HR (\%) }\end{array}$ & N/A & $-23 \pm 12$ & $-17 \pm 15$ & 0.091 \\
\hline
\end{tabular}

high-dose carvedilol showed significantly greater reductions in MAP $(-17 \pm 10$ vs $-11 \pm 13 \% ; \mathrm{p}=0.043)$ and HR $(-20 \pm 12$ vs $-12 \pm 15 \% ; \mathrm{p}=0.033)$ than patients receiving low-dose carvedilol treatment. However, the degree of HVPG reduction was similar in patients receiving high-dose and low-dose carvedilol treatment, respectively $(-20 \pm 10 \%$ vs $-18 \pm 12 \% ; \mathrm{p}=0.442)$.

\section{Bleeding rates during follow-up}

During a mean follow-up of 19.5 months, 13\% (14/104) of patients experienced an episode of variceal bleeding (figure $3 \mathrm{~A}$ and table 4). Significantly lower bleeding rates were observed in the haemodynamic responders (PROP/CARV) when compared to the EBL patients $(11 \% / 8 \%$ vs $24 \% ; \mathrm{p}=0.0429)$. Notably, no patient bled between enrolment and assignment to a treatment group. Among the propranolol non-responders, a significantly lower incidence of variceal bleeding was observed in the CARV arm compared to the EBL arm $(\mathrm{p}=0.0218)$.

\section{Hepatic decompensation and mortality}

Overall, 38\% (40/104) of patients showed hepatic decompensation during follow-up with ascites $(n=13)$, variceal bleeding $(n=11)$, hepatic encephalopathy $(n=8)$ and jaundice $(n=8)$ as reasons for hepatic decompensation (figure 3B,C and table 4). When comparing hepatic decompensation between haemodynamic responders and EBL-treated patients, a trend towards a 
Table 3 Effects of drug dosing on haemodynamic parameters

\begin{tabular}{llllllc}
\hline & N & MAP $(\%)$ & p Value & HR $(\%)$ & p Value & HVPG (\%) \\
\hline Propranolol (80-100 mg/day) & 67 & $-10 \pm 15$ & 0.328 & $-21 \pm 13$ & 0.106 & $-11 \pm 13$ \\
Propranolol (120-160 mg/day) & 27 & $-12 \pm 11$ & & $-27 \pm 9$ & & $-13 \pm 10$ \\
\hline Carvedilol (6.25-12.5 mg/day) & 40 & $-11 \pm 13$ & 0.043 & $-12 \pm 15$ & 0.023 & $-18 \pm 12$ \\
Carvedilol (25-50 mg/day) & 27 & $-17 \pm 10$ & & $-22 \pm 13$ & & $-20 \pm 10$ \\
\hline
\end{tabular}

Comparison of parameters among subgroups by Mann-Whitney $U$ test.

$H R$, heart rate; HVPG, hepatic venous pressure gradient; MAP, mean arterial pressure.

higher rate of hepatic decompensation was noted in the EBL group $(55 \%)$ than in the PROP $(38 \%)$ or CARV $(26 \%)$ groups $(\mathrm{p}=0.0789)$. Most, importantly among propranolol non-responders, carvedilol responders had a significantly lower incidence of hepatic decompensation than EBL-treated patients ( $26 \%$ vs $55 \% ; p=0.0351)$. The development of ascites $(p=0.031)$ and variceal bleeding $(\mathrm{p}=0.012)$ were significantly more common in the EBL group than in haemodynamic responders (PROP/ CARV). Overall, 9\% (9/104) of patients were diagnosed with HCC during follow-up, with no significant differences among the treatment groups.

Seventeen per cent (18/104) of patients died during follow-up and $9 \%$ (9/104) underwent liver transplantation. When comparing TFS among patients treated with propranolol, carvedilol, or EBL, respectively, TFS was significantly higher in the haemodynamic responders CARV/PROP than in the EBL group (mean 495 vs 417 days; $p=0.0455$ ). A trend towards longer TFS was noted in carvedilol responders compared to EBL-treated patients (mean 484 vs 417 days; $\mathrm{p}=0.0618$ ). The better survival in the PROP/CARV groups was mainly due to fewer bleeding-related and liver-related deaths, while deaths from other causes were observed with similar frequencies in the EBL group.

\section{DISCUSSION}

This is the first study reporting the efficacy of carvedilol for primary prophylaxis in cirrhotic patients not responding to
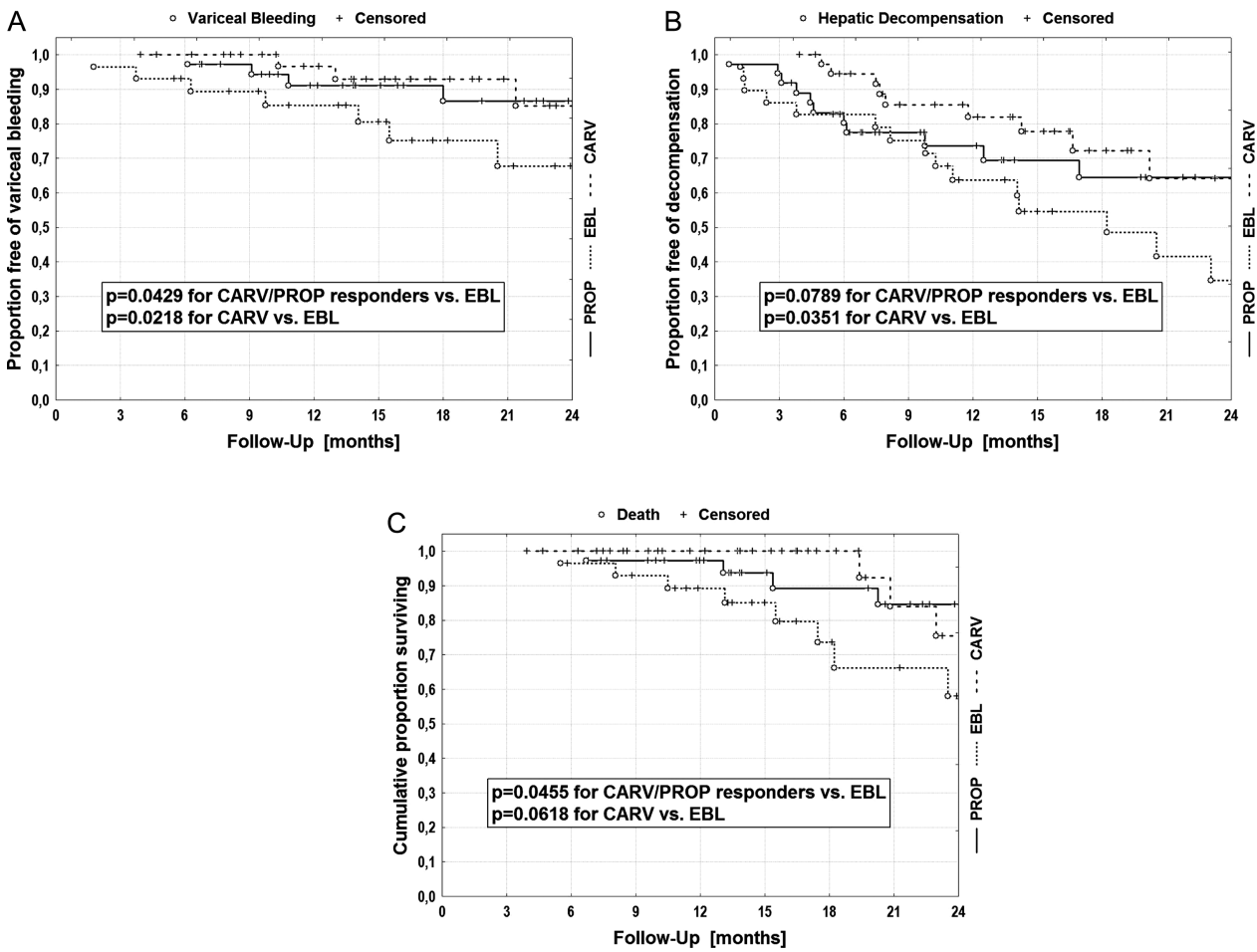

Figure 3 Bleeding rates, hepatic decompensation and survival. (A) This figure shows the proportion of patients remaining free of variceal bleeding by Kaplan-Meier plot. Subgroups treated with propranolol, carvedilol, or EBL for primary prophylaxis of variceal bleeding were compared by log-rank test. A significantly lower rate of variceal bleeding was seen in haemodynamic responders to propranolol and carvedilol when compared to patients treated with EBL. Among propranolol non-responders, bleeding rates were significantly lower in carvedilol responders than in the group treated by EBL. (B) This figure shows the proportion of patients remaining free of hepatic decompensation by Kaplan-Meier plot. Subgroups treated with propranolol, carvedilol, or EBL for primary prophylaxis of variceal bleeding were compared by log-rank test. A trend towards a lower rate of hepatic decompensation was seen in haemodynamic responders to propranolol and carvedilol when compared to patients treated with EBL. Among propranolol non-responders, hepatic decompensation rates were significantly lower in carvedilol responders than in the group treated by EBL. (C) This figure shows the TFS of patients by Kaplan-Meier plot. Subgroups treated with propranolol, carvedilol, or EBL for primary prophylaxis of variceal bleeding were compared by log-rank test. TFS was significantly longer in patients with haemodynamic response to propranolol and carvedilol than in patients treated with EBL. Among propranolol non-responders, a trend towards improved TFS was seen in carvedilol responders compared to patients treated by EBL. CARV, patients treated with carvedilol; EBL, endoscopic band ligation; PROP, patients treated with propranolol; TFS, transplant-free survival. 
Table 4 Outcome during follow-up

\begin{tabular}{|c|c|c|c|c|c|}
\hline Patients (n) & All 104 & $\begin{array}{l}\text { Propranolol responder } \\
37(35.6 \%)\end{array}$ & $\begin{array}{l}\text { Carvedilol responder } \\
38(36.5 \%)\end{array}$ & $\begin{array}{l}\text { Non-responder (EBL group) } \\
29(27.9 \%)\end{array}$ & p Value* \\
\hline Follow-up, months & $19.5 \pm 9.7$ & $20.9 \pm 9.2$ & $18.9 \pm 9.5$ & $19.5 \pm 10.4$ & 0.391 \\
\hline Variceal bleeding, n (\%) & $14(13 \%)$ & $4(11 \%)$ & $3(8 \%)$ & $7(24 \%)$ & 0.043 \\
\hline Hepatic decompensation, n (\%) & $40(38 \%)$ & $14(38 \%)$ & $10(26 \%)$ & $16(55 \%)$ & 0.079 \\
\hline Ascites & $13(12 \%)$ & $4(11 \%)$ & $4(10 \%)$ & $5(17 \%)$ & 0.031 \\
\hline HE grade III/IV & $8(7 \%)$ & $3(8 \%)$ & $2(5 \%)$ & $3(10 \%)$ & 0.148 \\
\hline Variceal bleeding & $11(10 \%)$ & $3(8 \%)$ & $2(5 \%)$ & $6(21 \%)$ & 0.012 \\
\hline Jaundice & $8(7 \%)$ & $4(11 \%)$ & $2(5 \%)$ & $2(7 \%)$ & 0.518 \\
\hline $\mathrm{HCC}, \mathrm{n}(\%)$ & $7(6 \%)$ & $3(8 \%)$ & $1(2 \%)$ & $3(10 \%)$ & 0.281 \\
\hline OLT, n (\%) & $9(9 \%)$ & $4(11 \%)$ & $2(5 \%)$ & $3(10 \%)$ & 0.412 \\
\hline TFS, days & $483 \pm 284$ & $504 \pm 273$ & $484 \pm 269$ & $417 \pm 218$ & 0.046 \\
\hline Death, n (\%) & $18(17 \%)$ & $5(14 \%)$ & $4(11 \%)$ & $9(31 \%)$ & 0.018 \\
\hline Bleeding related & $8(7 \%)$ & $2(5 \%)$ & $1(2 \%)$ & $5(17 \%)$ & $<0.01$ \\
\hline Liver related & $6(5 \%)$ & $2(5 \%)$ & $1(2 \%)$ & $3(10 \%)$ & 0.020 \\
\hline Other & $4(3 \%)$ & $1(2 \%)$ & $2(5 \%)$ & $1(3 \%)$ & 0.494 \\
\hline
\end{tabular}

*Statistical comparisons were performed by log-rank test.

EBL, endoscopic band ligation; HCC, hepatocellular carcinoma; HE, hepatic encephalopathy; OLT, orthotopic liver transplantation; TFS, transplant-free survival.

propranolol including data on long-term follow-up. We evaluated the responses rates to carvedilol in HVPG-documented non-responders to propranolol treatment and showed that a significant proportion of propranolol non-responders $(\sim 50 \%)$ can achieve a haemodynamic response to carvedilol treatment. Carvedilol had significantly greater effects on portal pressure than propranolol ( $-19 \%$ vs $-12 \%)$, while changes in MAP and in HR were not significantly different between propranolol and carvedilol-treated patients. In summary, approximately two-thirds (72\%) of our patients achieved a haemodynamic response to NSBB when carvedilol was used as 'rescue' treatment in propranolol non-responders. Approximately one-third $(28 \%)$ of patients did not achieve a haemodynamic response and were finally treated with EBL until eradication of varices.

When using only propranolol for prophylaxis of variceal bleeding, a substantial number of patients would have remained at higher risk of variceal bleeding, because haemodynamic response was only achieved in 39\%. Carvedilol was able to decrease the risk of bleeding substantially in propranolol non-responders, as a further reduction in portal pressure of $-13 \%$ could be achieved resulting in haemodynamic response rates of $56 \%$ among propranolol non-responders. Using this 'a la carte' approach of HVPG-guided NSBB therapy and EBL in pharmacological nonresponders, the overall rate of first variceal bleeding was low at $13 \%$ during a follow-up period of 2 years. Lower bleeding rates were observed in haemodynamic responders (PROP/CARV groups) than in haemodynamic non-responders (EBL group). Most importantly, carvedilol was significantly more effective in preventing variceal bleeding in propranolol non-responders than EBL, underlining that achieving a haemodynamic response is associated with better prognosis in the long term. ${ }^{6-8}$

In addition, we investigated potential differences in systemic haemodynamics between propranolol and carvedilol treatment. When using median doses of $100 \mathrm{mg} /$ day of propranolol and $12.5 \mathrm{mg} /$ day of carvedilol, no differences in MAP and HR were observed between the PROP and CARV groups, respectively. However, the doses of propranolol used in previous trials varied from $80 \mathrm{mg} /$ day to $320 \mathrm{mg} /$ day and were not consistent. As dose finding studies have not been performed to identify the 'best portal-hypotensive' dose for carvedilol in cirrhotic patients with PHT so far, the optimal dose needs to be defined. Higher doses of carvedilol-as with propranolol-may cause an even greater decrease in portal pressure, but systemic arterial hypotensive effects may limit the use of higher doses in the long run. Therefore, we used the currently recommended approach of titrating the NSBB dose according to $\mathrm{ABP}_{\text {sys }}$ and $\mathrm{HR}$, and compared the effects of different propranolol and carvedilol doses on systemic and portal haemodynamics. An increase of propranolol doses from $80-100 \mathrm{mg} /$ day to $120-160 \mathrm{mg} /$ day did not result in a significant further decrease of MAP, HR, or HVPG. In contrast, an increase of carvedilol doses from $6.25-12.5 \mathrm{mg} /$ day to $25-$ $50 \mathrm{mg}$ /day significantly further decreased MAP and HR without an additional effect on HVPG. These haemodynamic results support the use of low carvedilol doses of $6.25-12.5 \mathrm{mg} / \mathrm{day}$, because this would-at least to some degree-avoid adverse effects related to arterial hypotension or bradycardia.

The current recommendations ${ }^{9}$ to use either NSBB or EBL for primary prophylaxis of variceal bleeding are based on studies that did not assess the haemodynamic response to NSBB. Although it seems likely that a substantial number of NSBB non-responders were also treated with NSBB in those studies, NSBB showed an equal performance compared to EBL in terms of survival but were less effective for primary prophylaxis of variceal bleeding. ${ }^{26}$ Now having a more potent drug such as carvedilol that may lead to haemodynamic response in up to $72 \%$ of all treated cirrhotic patients, pharmacological prophylaxis may perform better than EBL, as suggested by the study of Tripathi et al. ${ }^{20}$

We also evaluated the incidence of hepatic decompensation and mortality during a 2-year follow-up period according to the treatment group. Overall, $38 \%$ of our patients experienced an episode of hepatic decompensation during follow-up. Haemodynamic responders to propranolol and carvedilol had a lower incidence of hepatic decompensation than the EBL group, while the difference was only significant when comparing carvedilol responders to EBL-treated patients. Interestingly, even TFS was better in the haemodynamic responders to propranolol and carvedilol than in the EBL group. NSBB may thus be an independent protective factor for hepatic decompensation, at least in patients with varices and haemodynamic response. Still, the potential adverse effects of long-term carvedilol treatment on systemic haemodynamics and renal perfusion have to be considered. ${ }^{19}$ In addition, a recent study reported increased mortality when using NSBB in cirrhotic patients with ascites. ${ }^{27}$ It seems that if NSBB are used in cirrhotic patients with varices (who have an indication for NSBB), achieving 
a haemodynamic response to the NSBB propranolol or carvedilol exerts beneficial effect in terms of reducing the risk of hepatic decompensation (including the development of ascites) and mortality. ${ }^{6-8}$ In our study, we were also able to demonstrate a favourable prognosis and improved survival in well-compensated patients with cirrhotic achieving a haemodynamic response to NSBB over a period of 2 years; however, the long-term survival rates of cirrhotic patients on continuous NSBB treatment should be investigated further.

One limitation of our study is the lack of randomisation at baseline or at the time of propranolol non-response, which may have introduced a bias in the study population. However, post-hoc comparisons of baseline characteristics revealed no significant differences in important prognostic baseline parameters in cirrhotic patients with PHT. Most importantly, the degree of PHT (HVPG) and the severity of liver disease (Child-Pugh score, model of end-stage liver disease) were similar among the PROP, CARV and EBL groups. The proportion of patients with large oesophageal varices was numerically higher in the EBL group than in the PROP/CARV groups, while the prevalence of red spots signs was higher in the PROP/CARV groups than in the EBL group. The exclusion of patients with severe liver failure and/or with hepatorenal syndrome may limit our findings to those patients with compensated liver disease, especially when considering the recent data ${ }^{27}$ on the potential detrimental effects of NSBB in patients with cirrhotic and refractory ascites.

'High-dose' NSBB treatment was better tolerated in the CARV group than in the PROP group, which may have contributed to the higher haemodynamic response rates in the CARV group. However, the cut-off between low and high doses was chosen empirically and no study to date has shown a clear correlation of NSBB dosing and rates of haemodynamic response. In addition, our comparison of dose and haemodynamic effects has shown that higher doses of carvedilol have more pronounced systemic haemodynamic effect (on MAP and on HR), but are not associated with a further decrease of portal pressure.

This study shows that carvedilol is a well-tolerated and highly effective drug for the pharmacological treatment of PHT. Carvedilol shows great efficacy in haemodynamic non-responders to propranolol resulting in a substantial increase in the proportion of haemodynamic responders, with up to $72 \%$ of medically treated patients achieving a HVPG response. Bleeding rates during a follow-up period of 2 years were significantly lower in haemodynamic responders to propranolol and carvedilol than in patients treated with EBL. Our data suggest that increasing the dose of carvedilol above $12.5 \mathrm{mg}$ may just increase unwanted systemic/renal side effects while lacking a greater portal-hypotensive effect. The optimal dose of carvedilol will have to be assessed in separate dosefinding studies. Most importantly, achieving a carvedilol response in propranolol non-responders is associated with a reduced risk of hepatic decompensation and increased TFS compared to EBL-treated propranolol non-responders.

Contributors TR, GU, AF, MPR: study concept and design. TR, GU, AF, BAP, PS, MP, BBH: acquisition of data. TR, GU, AF, MT, LK, MPR: analysis and interpretation of data. TR: drafting of the manuscript. TR, AF, MT, LK, MPR: critical revision of the manuscript.

Funding None.

Competing interests None.

Patient consent Obtained.

Ethics approval This study was conducted following the ethical principles of the revised Declaration of Helsinki and was approved by the ethics committee of the Medical University of Vienna.

Provenance and peer review Not commissioned; internally peer reviewed.

\section{REFERENCES}

1 Bosch J, Garcia-Pagan JC. Complications of cirrhotic. I. Portal hypertension. J Hepatol 2000;32:141-56.

2 Ferlitsch M, Reiberger T, Hoke M, et al. Von Willebrand factor as new non-invasive predictor of portal hypertension, decompensation and mortality in patients with liver cirrhotic. Hepatology 2012;124:395-402.

3 Garcia-Tsao G, Groszmann RJ, Fisher RL, et al. Portal pressure, presence of gastroesophageal varices and variceal bleeding. Hepatology 1985;5:419-24.

4 Groszmann RJ, Bosch J, Grace ND, et al. Hemodynamic events in a prospective randomized trial of propranolol versus placebo in the prevention of a first variceal hemorrhage. Gastroenterology 1990:99:1401-7.

5 Cerqueira RM, Andrade L, Correia MR, et al. Risk factors for in-hospital mortality in cirrhotic patients with oesophageal variceal bleeding. Eur J Gastroenterol Hepatol 2012:24:551-7.

6 Abraldes JG, Tarantino I, Turnes J, et al. Hemodynamic response to pharmacological treatment of portal hypertension and long-term prognosis of cirrhotic. Hepatology 2003;37:902-8

7 Merkel C, Bolognesi M, Sacerdoti D, et al. The hemodynamic response to medical treatment of portal hypertension as a predictor of clinical effectiveness in the primary prophylaxis of variceal bleeding in cirrhotic. Hepatology 2000;32: 930-4

8 D'Amico G, Garcia-Pagan JC, Luca A, et al. Hepatic vein pressure gradient reduction and prevention of variceal bleeding in cirrhotic: a systematic review. Gastroenterology 2006;131:1611-24.

9 de Franchis R. Revising consensus in portal hypertension: report of the Baveno V consensus workshop on methodology of diagnosis and therapy in portal hypertension. J Hepatol 2010;53:762-8.

10 Lo GH, Chen WC, Chen MH, et al. Banding ligation versus nadolol and isosorbide mononitrate for the prevention of esophageal variceal rebleeding. Gastroenterology 2002;123:728-34.

11 Garcia-Pagan JC, Morillas R, Banares R, et al. Propranolol plus placebo versus propranolol plus isosorbide-5-mononitrate in the prevention of a first variceal bleed: a double-blind RCT. Hepatology 2003;37:1260-6.

12 Schepke M, Kleber G, Nurnberg D, et al. Ligation versus propranolol for the primary prophylaxis of variceal bleeding in cirrhotic. Hepatology 2004;40:65-72.

13 Sarin SK, Lamba GS, Kumar M, et al. Comparison of endoscopic ligation and propranolol for the primary prevention of variceal bleeding. $N$ Engl J Med 1999;340:988-93

14 Bosch J. Carvedilol for portal hypertension in patients with cirrhotic. Hepatology 2010:51:2214-18.

15 Hemstreet BA. Evaluation of carvedilol for the treatment of portal hypertension. Pharmacotherapy 2004;24:94-104.

16 Tsochatzis EA, Triantos CK, Burroughs AK. Gastrointestinal bleeding: carvedilol-the best beta-blocker for primary prophylaxis? Nat Rev Gastroenterol Hepatol 2009:6:692-4.

17 Forrest EH, Bouchier IA, Hayes PC. Acute haemodynamic changes after oral carvedilol, a vasodilating beta-blocker, in patients with cirrhotic. J Hepatol 1996;25:909-15.

18 Banares R, Moitinho E, Piqueras B, et al. Carvedilol, a new nonselective beta-blocker with intrinsic anti-alpha1-adrenergic activity, has a greater portal hypotensive effect than propranolol in patients with cirrhotic. Hepatology 1999;30:79-83.

19 Banares R, Moitinho E, Matilla A, et al. Randomized comparison of long-term carvedilol and propranolol administration in the treatment of portal hypertension in cirrhotic. Hepatology 2002;36:1367-73.

20 Tripathi D, Ferguson JW, Kochar N, et al. Randomized controlled trial of carvedilol versus variceal band ligation for the prevention of the first variceal bleed. Hepatology 2009;50:825-33.

21 Peck-Radosavljevic M, Trauner M, Schreiber F. Austrian consensus on the definition and treatment of portal hypertension and its complications. Endoscopy 2005:37:667-73.

22 Reiberger $T$, Ferlitsch A, Payer BA, et al. Non-selective beta-blockers improve the correlation of liver stiffness and portal pressure in advanced cirrhotic. J Gastroenterol 2011;47:561-8.

23 Bosch J, Abraldes JG, Berzigotti A, et al. The clinical use of HVPG measurements in chronic liver disease. Nat Rev Gastroenterol Hepatol 2009;6:573-82.

24 Reiberger T, Rutter K, Ferlitsch A, et al. Portal pressure predicts outcome and safety of antiviral therapy in cirrhotic patients with hepatitis $C$ virus infection. Clin Gastroenterol Hepatol 2011;9:602-8 e1.

25 Reiberger T, Ferlitsch A, Payer BA, et al. Noninvasive screening for liver fibrosis and portal hypertension by transient elastography-a large single center experience. Wien Klin Wochenschr 2012;56:1439-47.

26 Imperiale TF, Chalasani N. A meta-analysis of endoscopic variceal ligation for primary prophylaxis of esophageal variceal bleeding. Hepatology 2001;33:802-7.

27 Serste T, Melot C, Francoz C, et al. Deleterious effects of beta-blockers on survival in patients with cirrhotic and refractory ascites. Hepatology 2010;52:1017-22. 